\title{
TASAS DE MORTALIDAD, ELIMINADOS Y DESCARTES DE DOS LÍNEAS GENÉTICAS DE POLLOS DE CARNE
}

\author{
Mortality, Removal, and Discarded Rates in Broilers of two Genetic Lines \\ María Francia M. ${ }^{1}$, Eliana Icochea D. ${ }^{1,2}$, Pablo Reyna S. ${ }^{1}$ y Edgardo Figueroa T. ${ }^{3}$
}

\section{RESUMEN}

\begin{abstract}
Se evaluó el porcentaje de mortalidad, eliminados y descartes de pollos de carne de dos líneas genéticas comerciales, criados en tres campañas consecutivas de 45 a 49 días cada una, bajo condiciones de manejo similares. Se usaron 360 aves de ambos sexos por línea genética (A y B) en cada campaña. Las aves eliminadas fueron aquellas que manifestaron severo retraso en el crecimiento, y las de descarte fueron aquellas con lesiones o anormalidades al momento de la saca. Las aves muertas y las eliminadas fueron necropsiadas. En ambas líneas, las principales causas de mortalidad fueron síndrome ascítico y síndrome de muerte súbita. La línea B tuvo mayor porcentaje de mortalidad en la $1^{\text {a }}$ campaña $(\mathrm{p}<0.05)$; sin embargo, no hubo diferencias estadísticas en los porcentajes de eliminados y de descarte entre líneas genéticas. La principal causa de descarte en ambas líneas fue problemas de patas, principalmente condrodistrofias. En términos generales, se puede concluir que la respuesta productiva en términos de mortalidad, eliminación y descartes fue similar en las dos líneas genéticas.
\end{abstract}

Palabras clave: líneas genéticas, pollos de carne, mortalidad, eliminado, descarte, campañas

\section{Abstract}

The present work evaluated the mortality, removal, and discarded rates in broilers of two commercial genetic lines, reared in three consecutive cycles of 45-49 days each under similar management conditions. In each cycle, 360 birds per genetic line (A and B) were used. Necropsy was carried out to dead and removed birds. Removed birds were those that showed severe growth delay and discarded birds were those that showed injuries or abnormalities at the end of the cycle. In both lines, the main causes of mortality were the ascites syndrome and sudden death syndrome. Line B showed higher mortality rate during the first cycle $(\mathrm{p}<0.05)$, but there was no statistical difference in the removal and discarded rates between the two genetics lines. The main cause for removing birds in both lines was leg problems, mainly chondrodystrophia. In general, it can be concluded that productive performance in terms of mortality, removing and discarding birds was similar between both genetic lines.

Key words: genetic lines, broilers, mortality, removed, discarded, cycle

${ }^{1}$ Laboratorio de Patología Aviar, ${ }^{3}$ Laboratorio de Producción Avícola, Facultad de Medicina Veterinaria, Universidad Nacional Mayor de San Marcos, Lima

${ }^{2}$ E-mail: eliana.icochea@gmail.com 


\section{INTRODUCCIÓN}

El sector avícola representa en la actividad agropecuaria el $57 \%$ del PBI pecuario. Además, aporta cerca del $70 \%$ de proteína animal consumida por la población nacional, en la forma de carne y huevos. La producción de carne de ave ha seguido una tendencia creciente en los últimos años, debido a su mayor oferta, facilidad de preparación y menor costo comparado con las carnes rojas de vacuno y ovino (MINAG, 2005).

La mejora genética de un ave conseguida por programas de selección, enfocados a una mayor ganancia de peso, conformación, producción de huevos y una mejor conversión alimenticia, ha alcanzado niveles sorprendentes (Solana, 1996). Sin embargo, el resultado de la selección genética en pollos de carne para lograr un elevado peso muscular en un marco óseo cada vez más pequeño, aunado al consumo de raciones altas en energía y proteína para satisfacer los nutrientes necesarios que demandan estas aves, ha generado un incremento en las tasas de mortalidad por enfermedades cardiovasculares y problemas del sistema músculoesquelético. También se ha hecho más frecuente el Síndrome de Hipertensión Pulmonar, causante de problemas comunes como la ascitis, y el Síndrome de Muerte Súbita (Julián y Díaz, 1999). Actualmente, la ascitis se considera como la enfermedad cardiovascular y metabólica más seria que enfrenta la industria avícola moderna (Maxwell y Robertson, 2001), además de otros problemas causantes de elevada mortalidad como la colibacilosis (Nepomuceno da Silva, 1998).

Las anormalidades del aparato locomotor se están presentando con más frecuencia por la alta velocidad de crecimiento (Salah, 2000). La incidencia y severidad parece variar entre líneas genéticas (Balkar, 1994). Estas anormalidades son consecuencia de alteraciones musculares, esqueléticas y nerviosas, que causan cojeras y dolor, retraso en el crecimiento, aumento de mortalidad y conversiones alimenticias pobres; y por lo tanto, ocasionan pérdidas económicas importantes por la cantidad de aves que se tienen que eliminar o descartar (Caamaño, 2000). Además, en los últimos años se ha visto un aumento de aves con retraso en el crecimiento que puede estar relacionado con la calidad del pollo, condiciones de manejo o problemas sanitarios que se observan con mayor incidencia durante los meses de invierno. Estas aves no alcanzan el tamaño adecuado en el tiempo esperado, causando problemas en las ventas y comercialización (Lott y Donald, 2003).

La avicultura se encuentra en una evolución constante a través de mejoras genéticas y nutricionales, mejorando los índices de viabilidad, ganancia de peso, eficiencia alimenticia y rendimiento de carcasa. Estas mejoras genéticas se observan con diferente potencial en las diversas líneas genéticas de pollos de carne (Yurika et al., 1994). Por este motivo, el presente estudio tuvo por objetivos la evaluación de las tasas de mortalidad, eliminados y descartes de dos líneas genéticas de pollos de carne, criados en tres campañas bajo condiciones de ambiente natural.

\section{Materiales y Métodos}

\section{Campañas y Aves}

El trabajo se realizó en el galpón de Producción Avícola de la Facultad de Medicina Veterinaria de la Universidad Nacional Mayor de San Marcos, Lima. El estudio comprendió tres subsiguientes ciclos de crianza de 45 a 49 días que se realizaron entre marzo y abril 2004, junio a julio 2004 y mayo a julio 2005.

Se emplearon 720 pollos de carne de un día de edad por campaña, correspondiendo 360 pollos para cada línea genética (A y B) y una distribución de 170 machos y 190 hembras por línea. 


\section{Diseño Experimental}

Cada línea genética fue considerada como un grupo experimental. En cada tratamiento se usaron 5 repeticiones de 34 machos y 5 de 38 hembras. Todas las aves recibieron el mismo manejo. El alimento y el agua se administraron ad libitum.

\section{Parámetros Evaluados}

Mortalidad. Se registró la mortalidad diaria y semanal. Se realizó la necropsia a las aves muertas en busca de hallazgos patológicos para determinar la causa de muerte.

Eliminados. Aves que manifestaron severos signos clínicos o anormalidades y que no mostraron capacidad de recuperación para competir con el resto de la población. También se realizó la necropsia a estas aves para determinar la causa exacta del problema que originó su eliminación.

Descartes. Aves que al momento de la comercialización en granja presentaron cualquier lesión o malformación (tal como cojeras u otros defectos físicos). Estos casos no fueron problemas de lote sino que constituyeron casos individuales.

\section{Análisis Estadístico}

La mortalidad, las aves eliminadas y los descartes se expresaron en porcentaje e intervalo de confianza (IC) al 95\%. La comparación entre porcentajes se basó en los respectivos intervalos de confianza.

\section{Resultados}

En el Cuadro 1 se muestra la frecuencia de casos de mortalidad, de aves eliminadas y descartadas durante las tres campañas, para las líneas genéticas A y B. Solamente se encontró diferencias estadísticas $(\mathrm{p}<0.05)$ por mortalidad de aves entre líneas genéticas en la primera campaña. Los porcentajes de eli- minados y de descartes no fueron estadísticamente diferentes entre las dos líneas.

La principal causa de mortalidad para ambas líneas genéticas fue el síndrome ascítico (Cuadro 2). En las tres campañas se pudo observar que la eliminación de aves ocurrió a partir de la $4^{\mathrm{a}}$ semana de edad hasta el término del experimento. La principal causa de eliminación en ambas líneas genéticas fue por problemas de patas y la segunda por severo retraso de crecimiento. La línea genética $B$ presentó mayor frecuencia de cojeras que determinaron la eliminación de estas aves, pero estas diferencias no fueron estadísticamente diferentes (Cuadro 3).

En ambas líneas genéticas, la principal causa de descarte fue por problemas de patas o "cojeras", seguido por retraso de crecimiento y buche penduloso (Cuadro 4).

\section{Discusión}

La mortalidad observada en el estudio correspondió mayormente a problemas derivados del rápido crecimiento, que son comunes en la crianza de broilers. No se encontró diferencias estadísticas en el porcentaje de mortalidad entre las dos líneas genéticas, coincidiendo con resultados de Stringhini et al. (2003) que mostraron que los pollos de carne de las líneas Ross, Lohmann y Arbor Acres presentaron desempeños semejantes. Asimismo, Moreira et al. (2004) tampoco encontraron diferencia estadística en porcentaje de mortalidad entre las líneas genéticas Ross 308, Cobb 500 e Hybro PG.

En la primera campaña, la mortalidad por ascitis fue menor comparada con la segunda y tercera campaña, posiblemente debido a la estación de verano en que se realizó esta crianza, época que permite manejar una mejor ventilación de los gallineros en comparación a las épocas de otoño e invier- 
Cuadro 1. Porcentajes de mortalidad, eliminadas y descartes en pollos de carne, de ambos sexos, de dos líneas genéticas

\begin{tabular}{llcc}
\hline \multirow{2}{*}{ Variable } & Campaña & \multicolumn{2}{c}{ Línea Genética } \\
\cline { 3 - 4 } & Primera & $1.39 \pm 1.21^{\mathrm{a}}$ & $5.00 \pm 2.25^{\mathrm{b}}$ \\
\hline \multirow{2}{*}{ Mortalidad (\%) } & Segunda & $2.50 \pm 1.61^{\mathrm{a}}$ & $2.22 \pm 1.52^{\mathrm{a}}$ \\
& Tercera & $3.33 \pm 1.85^{\mathrm{a}}$ & $6.66 \pm 2.57^{\mathrm{a}}$ \\
& Primera & $0.28 \pm 0.54^{\mathrm{a}}$ & $0.56 \pm 0.77^{\mathrm{a}}$ \\
Eliminadas (\%) & Segunda & 0 & $0.56 \pm 0.77$ \\
& Tercera & $2.78 \pm 1.70^{\mathrm{a}}$ & $3.06 \pm 1.78^{\mathrm{a}}$ \\
& Primera & $1.67 \pm 1.32^{\mathrm{a}}$ & $1.94 \pm 1.42^{\mathrm{a}}$ \\
Descarte (\%) & Segunda & $2.78 \pm 1.70^{\mathrm{a}}$ & $1.39 \pm 1.21^{\mathrm{a}}$ \\
& Tercera & $4.44 \pm 2.13^{\mathrm{a}}$ & $7.22 \pm 2.67^{\mathrm{a}}$ \\
\hline
\end{tabular}

${ }^{a, b}$ Superíndices diferentes entre líneas genéticas dentro de campañas indican diferencia estadística $(p<0.05)$.

Cuadro 2. Causas de mortalidad (\%) por campaña en pollos de carne de ambos sexos de dos líneas genéticas

\begin{tabular}{lcccccc}
\hline \multirow{2}{*}{ Causa } & \multicolumn{2}{c}{ Primera campaña } & \multicolumn{2}{c}{ Segunda campaña } & \multicolumn{2}{c}{ Tercera campaña } \\
\cline { 2 - 7 } & $\mathrm{A}$ & $\mathrm{B}$ & $\mathrm{A}$ & $\mathrm{B}$ & $\mathrm{A}$ & $\mathrm{B}$ \\
\hline Asfixia & 0.28 & 0 & 0 & 0.28 & 0 & 0.28 \\
$\begin{array}{l}\text { Onfalitis } \\
\begin{array}{l}\text { Colibacilosis de } \\
\text { origen }\end{array}\end{array}$ & 0 & 0.56 & 0 & 0 & 0.83 & 0 \\
$\begin{array}{l}\text { respiratorio } \\
\text { Trauma }\end{array}$ & 0.28 & 1.67 & 0.28 & 1.11 & 0.28 & 0.56 \\
$\begin{array}{l}\text { Ascitis- } \\
\text { hidropericardio }\end{array}$ & 0.28 & 0.56 & 1.67 & 0.56 & 1.12 & 3.61 \\
Muerte súbita & 0.28 & 1.95 & 0 & 0 & 0.84 & 1.95 \\
Ruptura de buche & 0.28 & 0 & 0.28 & 0 & 0 & 0 \\
Falsa deglución & 0 & 0 & 0.28 & 0 & 0 & 0 \\
Torsión intestinal & 0 & 0 & 0 & 0 & 0 & 0.28 \\
\hline Total & 1.39 & 5.00 & 2.5 & 2.22 & 3.33 & 6.66 \\
\hline
\end{tabular}


Cuadro 3. Causas de eliminación (\%) por campaña en pollos de carne de ambos sexos de dos líneas genéticas

\begin{tabular}{lcccccc}
\hline \multirow{2}{*}{ Causa } & \multicolumn{2}{c}{ Primera campaña } & \multicolumn{2}{c}{$\begin{array}{c}\text { Segunda } \\
\text { campaña }\end{array}$} & \multicolumn{2}{c}{ Tercera campaña } \\
\cline { 2 - 7 } & $\mathrm{A}$ & $\mathrm{B}$ & $\mathrm{A}$ & $\mathrm{B}$ & $\mathrm{A}$ & $\mathrm{B}$ \\
\hline $\begin{array}{l}\text { Retraso de tamaño } \\
\text { Problemas de patas o } \\
\text { cojeras }\end{array}$ & 0.28 & 0.28 & 0 & 0 & 0.56 & 0.56 \\
$\quad \begin{array}{l}\text { Osteocondrosis } \\
\text { Condrodistrofia } \\
\quad \text { Deformación } \\
\text { varus o valgus }\end{array}$ & 0 & 0.28 & 0 & 0 & 0.56 & 1.39 \\
\hline Total & 0 & 0 & 0 & 0.56 & 1.67 & 1.11 \\
\hline
\end{tabular}

Cuadro 4. Causas de descartes (\%) por campaña en pollos de carne de ambos sexos de dos líneas genéticas

\begin{tabular}{|c|c|c|c|c|c|c|}
\hline \multirow{2}{*}{ Causa } & \multicolumn{2}{|c|}{ Primera campaña } & \multicolumn{2}{|c|}{ Segunda campaña } & \multicolumn{2}{|c|}{ Tercera campaña } \\
\hline & A & B & A & $\mathrm{B}$ & A & B \\
\hline Retraso & 0 & 0 & 0.56 & 0.56 & 1.39 & 2.78 \\
\hline Buche penduloso & 0.83 & 1.67 & 0.83 & 0.28 & 0.28 & 0.28 \\
\hline $\begin{array}{l}\text { Problemas de } \\
\text { patas o cojeras }\end{array}$ & 0.83 & 0.28 & 0.83 & 0.56 & 2.78 & 4.17 \\
\hline Cuello torcido & 0 & 0 & 0.28 & 0 & 0 & 0 \\
\hline Canibalismo & 0 & 0 & 0.28 & 0 & 0 & 0 \\
\hline Total & 1.67 & 1.94 & 2.78 & 1.39 & 4.44 & 7.22 \\
\hline
\end{tabular}

no. Además, el consumo de alimento fue menor en las aves expuestas a temperaturas por encima de $25^{\circ} \mathrm{C}$ (verano), reduciendo la ganancia de peso y por lo tanto, una menor predisposición a la muerte por ascitis (Quintao et al., 1995).

La mayor causa de mortalidad en las tres campañas fue por síndrome ascítico, con la única excepción de la primera campaña para la línea genética A donde no hubo una causa que destaque en relación a las demás.
No se observó mayor variabilidad entre sexos, aunque en la tercera campaña se observó una mayor mortalidad por síndrome ascítico en machos, posiblemente debido a que estos crecen más rápido que las hembras. Walter (1993) reportó que la ascitis ocurre más frecuentemente en aves de crecimiento rápido, siendo los machos las aves más afectadas (hasta el 70\%) debido a la mayor velocidad de crecimiento que las hembras y la mayor demanda de una buena calidad del medio ambiente del gallinero. 
Las anormalidades en el aparato locomotor se consideran como uno de los factores principales que causan pérdidas económicas importantes. Según Thorp (1994), la presión de selección genética para una mayor tasa de crecimiento ha impuesto excesivas exigencias sobre la integridad del esqueleto en las líneas modernas de carne. La alteración del proceso normal de crecimiento óseo y la homeostasis han provocado enfermedades óseas que son evidentes en la industria avícola moderna. Por otro lado, Lott y Donald (2003) refieren que el amoniaco, por ser un gas irritante y nocivo, retrasa el crecimiento de las aves, especialmente en los meses más fríos cuando los galpones están herméticamente cerrados, conduciendo a problemas de eliminación y descartes de aves retrasadas. Estos problemas se notaron mayormente en la $3^{\mathrm{a}}$ campaña, estudio que se llevó a cabo en la época de invierno, donde la línea B obtuvo el mayor porcentaje (2.78\%) de descartes.

El buche penduloso fue otra de las causas importantes para el descarte de las aves. Esta anomalía parece obedecer a una distensión excesiva de la musculatura lisa del buche por el sobrellenado con alimento, causando ruptura de algunas fibras de elastina inmersas en las capas musculares del órgano, y puede, además, haber una predisposición genética de algunas líneas que son más susceptibles que otras (Farmer et al., 1990). Según Whiteman y Bickford (1983), casos esporádicos pueden estar relacionados con la parálisis del nervio vago. Esta anormalidad se registró mayormente en la línea genética B $(1.67 \%)$ durante la $1^{\mathrm{a}}$ campaña.

\section{Conclusiones}

La respuesta productiva en términos de mortalidad, eliminación y descartes fue similar en las dos líneas genéticas de producción de carne.

\section{Literatura Citada}

1. Balkar S. 1994. Broilers suffer from discondroplasia and femoral necrosis. World Poultry 10(10): 109-111.

2. Caamaño GJ. 2000. Problemas locomotores en aves. [Internet], [15 julio 2005]. Disponible en:www.criaderosloscipreses.com/gallos

3. Farmer $H$, Jones $R C$, Wilding GP, Jordan FT, Gooderham KR, Stuart JC. 1990. Miscellaneous conditions: pendulous crop. In: Poultry Diseases. $3^{\text {rd }}$ ed. London: Baillére Tindal Ed. p 362-363.

4. Julian R, Diaz G. 1999. La fisiología del pollo de engorde del futuro. Mundo Avícola y Porcino 32: 12-13.

5. Lott B, Donald J. 2003. El amoniaco puede causar pérdidas importantes. Ind Avícola 50(10): 8-10.

6. Maxwell M, Robertson G. 2001. Encuesta mundial de ascitis: parte 2. Ind Avícola 48(10): 16-27.

7. MINAG. 2005. Producción pecuaria: Aves. [Internet], [15 agosto 2005]. Disponible en: www.portalagrario.gob.pe/ pec_real.shtml

8. Moreira J, Mendes A, De Oliveira R, Garcia E, Alentar I, Garófallo R, Lima I. 2004. Efeito da densidade populacional sobre desempenho, rendimento de carcaça e qualidade da carne em grangos de corte de diferentes linhagens comerciais. Rev Brasileira Zootec 33(6): 1506-1519.

9. Nepomuceno da Silva E. 1998. Passo a passo contra E. coli. Avicultura Ind 1057: 24-26.

10. Quintao G, Da Silva D, De Almeida M, Brandao J. 1995. Desempenho comparativo de marcas comerciais e de cruzamentos de diferentes linhagens de frangos de corte produzidos na UFV, em diferentes niveis de energia. Consumo de raçao, ganho de peso e conversao alimentar. Rev Bras Zootec 24(5): 759767. 
11. Salah HM. 2000. New concepts of intestinal digestión and absorption in chickens. World Poultry 16(2): 14-16.

12. Solana AA. 1996. Significación patológica actual del virus de la enfermedad de Marek. [Internet], [15 agosto 2005]. Disponible en: www.racve.es/muestra actividad.php.

13. Stringhini JE, Laboissiere M, Muramatsu K, Mogyca N, Barcellos M. 2003. Avaliaçao do desempenho e rendimento de carcaça de quatro linhagens de frangos de corte criadas en Goias. Rev Bras Zootec 32(1): 183-190.
14. Thorp B. 1994. Transtornos óseos de las aves. Ind Avícola 41(1): 22-24.

15. Walter R. 1993. Un nuevo concepto en la fisiología de ascitis. Ind Avícola 40(2): 8-10.

16. Whiteman CE, Bickford AA. 1983. Manual de las enfermedades de las aves. $2^{\mathrm{a}}$ ed. Pennsylvania: Asociación Americana de Patólogos Aviares. 257 p.

17. Yurika J, Nicolao N, Waine J. 1994. Desempenho de duas linhagens comerciais de frangos de corte, criadas sob diferentes densidades populacionais e diferentes tipos de camas. Rev Bras Zootec 23(3): 476-484. 